\title{
Pediatric functional hemispherectomy: operative techniques and complication avoidance
}

\author{
Christopher C. Young, MD, PhD,1 John R. Williams, MD, ${ }^{1}$ Abdullah H. Feroze, MD, ${ }^{1}$ \\ Margaret McGrath, MD, ${ }^{1}$ Ali C. Ravanpay, MD, PhD, ${ }^{1,3}$ Richard G. Ellenbogen, MD, ${ }^{1,2}$ \\ Jeffrey G. Ojemann, MD, ${ }^{1,2}$ and Jason S. Hauptman, MD, PhD ${ }^{1,2}$
}

\begin{abstract}
1Department of Neurological Surgery, University of Washington; 'Department of Neurological Surgery, Seattle Children's Hospital; and ${ }^{3}$ Department of Neurological Surgery, VA Puget Sound Healthcare System, Seattle, Washington

Functional hemispherectomy/hemispherotomy is a disconnection procedure for severe medically refractory epilepsy where the seizure foci diffusely localize to one hemisphere. It is an improvement on anatomical hemispherectomy and was first performed by Rasmussen in 1974. Less invasive surgical approaches and refinements have been made to improve seizure freedom and minimize surgical morbidity and complications. Key anatomical structures that are disconnected include the 1) internal capsule and corona radiata, 2) mesial temporal structures, 3) insula, 4) corpus callosum, 5) parietooccipital connection, and 6) frontobasal connection. A stepwise approach is indicated to ensure adequate disconnection and prevent seizure persistence or recurrence. In young pediatric patients, careful patient selection and modern surgical techniques have resulted in $>80 \%$ seizure freedom and very good functional outcome. In this report, the authors summarize the history of hemispherectomy and its development and present a graphical guide for this anatomically challenging procedure. The use of the osteoplastic flap to improve outcome and the management of hydrocephalus are discussed.
\end{abstract}

https://thejns.org/doi/abs/10.3171/2020.1.FOCUS19889

KEYWORDS epilepsy surgery; hemispherectomy; hemispherotomy; osteoplastic flap

$\mathrm{F}$ UNCTIONAL hemispherectomy/hemispherotomy is a disconnection surgery that has undergone significant evolution. Today, when performed in high-volume epilepsy centers in appropriate patients, it is an effective procedure for the control of unilateral medically refractory epilepsy. Surgical modifications and improvements have resulted in excellent seizure outcome with low operative morbidity and mortality.

In 1938, McKenzie presented the first report of anatomical hemispherectomy for the treatment of refractory epilepsy at the annual meeting of the American Medical Association in San Francisco. The title of his report was quite simply, "The present status of a patient who had the right cerebral hemisphere removed." ${ }^{15}$ Following removal of the right cerebral hemisphere in a patient with spastic hemiparesis, the seizures stopped. In 1950, working in Johannesburg, South Africa, Krynauw reported on 12 patients with infantile hemiplegia who underwent hemispherectomy; seizure control was achieved in 10 of these patients, with improvement in function and behavior. ${ }^{12}$ This pioneering work led to a wave of enthusiasm for the procedure. By 1961, a review of the literature revealed 269 reported cases, with an operative mortality of $6.6 \% .^{24}$ Although the effectiveness of hemispherectomy was established, the high incidence of hydrocephalus and delayed mortality from superficial cerebral hemosiderosis in up to one-third of patients led to a rapid decline in the procedure. ${ }^{17,25}$ In the 1970s, Rasmussen recognized that the extent of resection and the residual surgical cavity were contributing factors to superficial cerebral hemosiderosis. Preservation of the frontal and occipital lobes and disconnecting them from the rest of the brain resulted in a "functional complete but anatomical subtotal hemispherectomy," giving rise to the functional hemispherectomy, which protected against superficial cerebral hemosiderosis and delayed hydrocephalus, and to a resurgence for the disconnection procedure. ${ }^{18}$ Subsequent modifications of the functional hemispherectomy have resulted in smaller craniotomies and the development of hemispherotomies, which have minimized the amount of brain tissue resected and operative time,

ABBREVIATIONS ACA = anterior cerebral artery; $A E D$ = antiepileptic drug; $E E G$ = electroencephalography; $E V D$ = external ventricular drain; fMRI = functional MRI; IONM $=$ intraoperative neurophysiological monitoring; $\mathrm{MCA}=$ middle cerebral artery.

SUBMITTED December 1, 2019. ACCEPTED January 28, 2020.

INCLUDE WHEN CITING DOI: 10.3171/2020.1.FOCUS19889. 
thereby reducing operative morbidity and mortality while maintaining an equivalent rate of seizure control. ${ }^{6,11,19,20,23}$

In this paper, we discuss the multidisciplinary evaluation and preoperative workup that is prerequisite prior to disconnection surgery at Seattle Children's Hospital. We present the surgical technique for a modified functional hemispherectomy/hemispherotomy, which combines elements of the peri-insular hemispherotomy described by Villemure and Daniel and the vertical parasagittal hemispherotomy described by Delalande et al., ${ }^{1,6,21}$ In addition, we review outcomes following modern disconnection surgery and discuss strategies to prevent common complications such as incomplete disconnection, the use of an osteoplastic flap, and the prevention of delayed hydrocephalus.

\section{Indication and Preoperative Workup Indications}

In order to be considered for operative intervention, patients must have a medically refractory, severe, disabling seizure disorder. ${ }^{7}$ They must have undergone satisfactory trials of tolerable antiepileptic drugs (AEDs) for at least 1 year. Medically refractory seizures are considered after failure of high-dose monotherapy with 2 distinct AEDs and 1 attempt at polytherapy. Conditions that may be amenable to a hemispherectomy are characterized by diffuse damage lateralized to one hemisphere that results in medically refractory epilepsy. These categories are typically grouped into acquired (e.g., perinatal cerebral infarction or intracranial hemorrhage, hemiconvulsion-hemiplegia epilepsy syndrome, hemimegalencephaly, hemispheric cortical dysplasia) or progressive (e.g., Rasmussen's encephalitis, Sturge-Weber syndrome) etiologies.,10

\section{Preoperative Workup}

At our institution, there is a stepwise approach to the preoperative evaluation by the multidisciplinary epilepsy team (Fig. 1). The first phase consists of the following: 1) clinical history and examination, which include semiology, history of drug resistance, other pertinent family orpersonal medical history, and neurological examination; 2) neurophysiology testing, with noninvasive video electroencephalography (EEG) being the primary modality, but optional additional modalities include dense array electrical source imaging or magnetoencephalography and magnetic source imaging; 3) anatomical imaging with an epilepsy-protocol MRI; 4) functional neuroimaging with FDG-PET or functional MRI (fMRI); and 5) neuropsychological testing. Phase 2 consists of intracranial EEG monitoring. Phase 3 consists of the surgery.

Depending on age and level of language development, the dominant hemisphere for language should be localized. $^{22}$ The preferred method is fMRI; however, it can also be done using the Wada test, an invasive intracarotid Amytal injection. In young pediatric patients who are severely disabled by refractory epilepsy, the affected hemisphere often has limited function, and there is a significant scope for postoperative neuroplasticity and compensation of function. Nevertheless, determination of functional localization preoperatively is useful to help with functional prognostication and counseling of the patient and parents.

If there is concordance of clinical epilepsy semiology, anatomical MRI abnormality, and clinical neurophysiology, then the next step is to move forward with surgery. If there is significant uncertainty regarding the localization of the seizure origin, phase 2 intracranial EEG may be necessary to obtain further information prior to disconnection surgery.

\section{Surgical Technique Anesthesia}

Anesthesia considerations are critical to the success of the procedure, taking into account the young age and small body size of the typical patient. If intraoperative neurophysiological monitoring (IONM) is used, patients are placed under total intravenous anesthesia to facilitate somatosensory evoked potentials and motor evoked potentials. Although not routinely used at our institution during hemispherectomy, IONM can provide information regarding the function of the contralateral hemisphere, particularly during corpus callosotomy where there is risk to structures in the contralateral hemisphere. During dissection of the mesial temporal structures, IONM potentially informs resection that endangers the midbrain and brainstem structures. Blood loss is closely monitored with red-packed cells available in the operating room, given the small circulating volume of the young pediatric patients undergoing disconnection procedures. For example, a 1-year-old child weighing $10 \mathrm{~kg}$ has a blood volume of $800 \mathrm{ml}$. A significant percentage of the circulating volume may be lost during the procedure, and constant vigilance and replacement management are indicated.

\section{Patient Positioning}

The patient is positioned supine with a small shoulder bump and the head turned to the contralateral side. The temporal lobe should be at the apex of the operative field and parallel to the floor (Fig. 2A and B). In patients younger than 2 years, the Infinity Mayfield head holder (Integra) is used. The head is immobilized by a low-pressure skull clamp (approximately $18 \mathrm{lb}$ ) with additional support from a horseshoe. The system provides superior stability and permits the use of optical neuronavigation. Neuronavigation is very useful for confirming anatomical landmarks and ensuring that the midline is not breached during corpus callosotomy, as well as confirming the extent of the frontobasal and parietooccipital disconnections.

\section{Osteoplastic Craniotomy}

After injection of lidocaine with $1 \%$ epinephrine, a standard question mark skin incision is made. Raney clips are applied for hemostasis. The skin flap is retracted above the superficial fascia until the frontal fat pad appears. A subfascial dissection (Fig. 2C) through the deep temporalis fascia is performed and elevated with the skin flap anteriorly to preserve frontal branches of the facial nerve, until the keyhole is adequately exposed. A large frontotemporal craniotomy is made, with the attachment of the temporalis muscles left intact over the zygoma (Fig. 2D). 


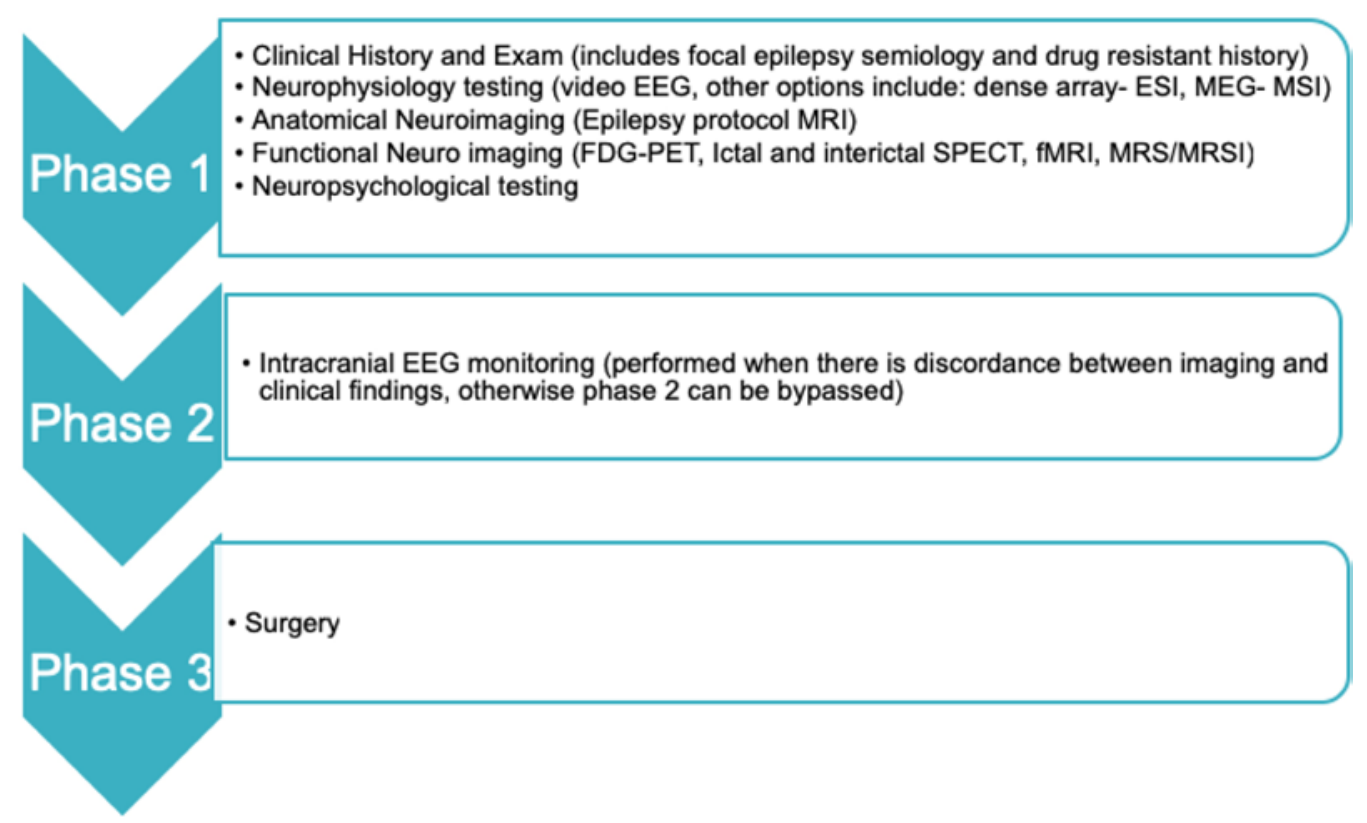

FIG. 1. Phases of epilepsy surgery. Protocol for preoperative evaluation for epilepsy surgery at Seattle Children's Hospital. Phase 1 is performed on all patients and consists of obtaining clinical, radiographic, and neurophysiological data. Phase 2 is performed only when there is discordance between imaging and clinical findings. Phase 3 includes operative planning and surgery. ESI = electric source imaging; MEG = magnetoencephalography; MRS = MR spectroscopy; MRSI = MR spectroscopic imaging; $M S I$ = magnetic source imaging.

This osteoplastic flap is kept out of the operative field with a lap towel and rubber bands (Fig. 2E and F). The dura is opened, and the dural edges are tacked up to reduce runoff into the surgical cavity and prevent epidural collections.

\section{Lateral Disconnection}

To disconnect the fibers forming the internal capsule and corona radiata, a corticectomy is performed in the middle frontal gyrus into the frontal lateral horn of the lateral ventricle (Fig. 3A and C). A cottonoid is immediately placed on the foramen of Monro to prevent blood from entering the contralateral hemisphere, reducing the risk of hydrocephalus. The corticectomy is extended posteriorly to the supramarginal gyrus following the course of the lateral ventricle into the atrium, where it is turned inferiorly toward the temporal horn (Fig. 3B and G). To facilitate surgical access, the frontal and temporal opercula are resected to expose the insula (Fig. 3D). The insular cortex is resected in a plane, which preserves the basal ganglia structures (Fig. 3E, F, and H). The middle cerebral artery (MCA) blood supply to the resected cortex is taken as needed to minimize blood loss, while supply to the frontal and parietal occipital lobes is preserved to prevent excessive tissue ischemia.

\section{Mesial Temporal Resection}

The anterior temporal lobe is removed, paying careful attention to the medial pial plane adjacent to the midbrain/ brainstem. When resection is complete, the oculomotor nerve and MCA bifurcation (limen insulae) can be visualized on the other side of the pia (Fig. 4A-C). The subpial resection ensures that the midbrain/brainstem is not inadvertently entered. Attention is turned to the temporal horn where the hippocampus and choroid fissure are visualized. The choroid fissure acts as an important landmark; structures medial to the fissure should not be violated, as they form part of the basal ganglia and thalamus. The inferior choroidal point is identified by the presence of the choroid plexus and the entry point of the anterior choroidal artery into the temporal horn. A line that joins the inferior choroidal point and the MCA bifurcation marks the extent of the amygdalectomy (Fig. 4D and E). The hippocampectomy is performed and taken to the tail.

\section{Transventricular Corpus Callosotomy}

The septum pellucidum is identified in the lateral ventricles, and the corpus callosotomy is commenced millimeters lateral to the midline (Fig. 5A and B). Care must be taken not to venture into the contralateral hemisphere. Anatomically, pericallosal branches of the anterior cerebral artery (ACA) and the falx serve as landmarks (Fig. $5 \mathrm{~B}, \mathrm{D}$, and $\mathrm{G}$ ) and can be confirmed with neuronavigation. The callosotomy is taken posteriorly through the body to the splenium until the atrium and the tentorial edge are reached. Anteriorly, the dissection follows the ACAs past the genu of the corpus callosum and courses inferiorly and posteriorly through the rostrum. The dissection is stopped approximately $5 \mathrm{~mm}$ anterior to the foramen of Monro in the coronal plane to prevent injury to the hypothalamus.

\section{Frontobasal and Parietooccipital Disconnections}

After completion of the callosotomy, posteriorly, the 

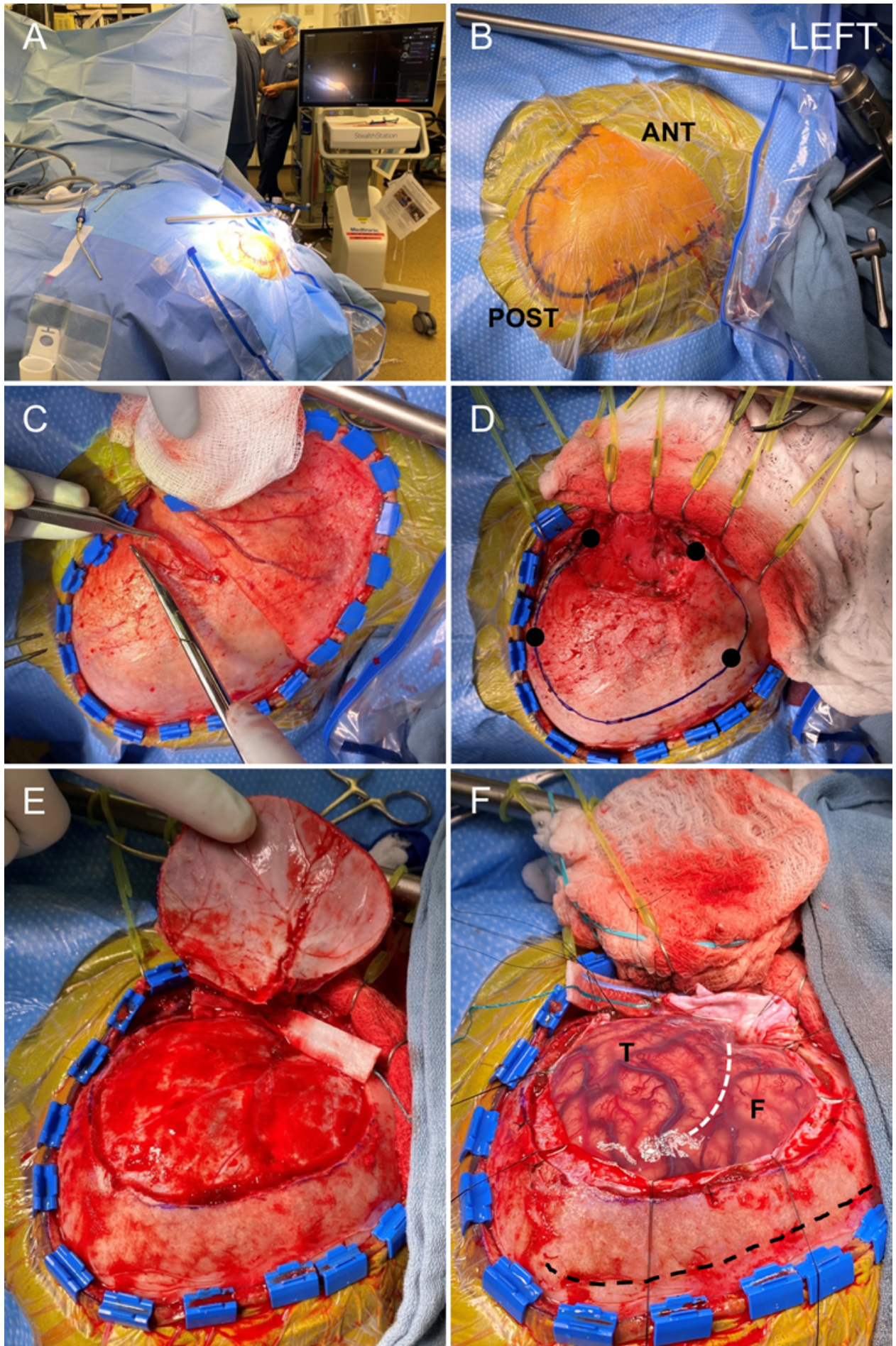

FIG. 2. Operative setup and left-sided osteoplastic craniotomy. A and B: The patient is positioned supine with the head turned to the contralateral side. The temporal lobe should be at the apex of the operative field and parallel to the floor. In patients younger than 2 years, the Infinity Mayfield head holder is used to immobilize the skull. Optical neuronavigation is used, and the monitor is placed at the head of the bed. Ant = anterior; post = posterior. C: The skin flap is reflected above the superficial fascia until the frontal fat pad appears. A subfascial dissection through the deep temporalis fascia is performed and elevated with the skin flap anteriorly to preserve frontal branches of the facial nerve. D and E: A large frontotemporal craniotomy is performed while preserving the overlying temporalis muscle attachments. F: The dura is opened, and the dural edges are tacked up to reduce runoff into the surgical cavity and prevent epidural collections. The locations of the frontal lobe $(\mathrm{F})$, temporal lobe $(\mathrm{T})$, sylvian fissure (white dotted line), and midline (black dotted line) are shown. 

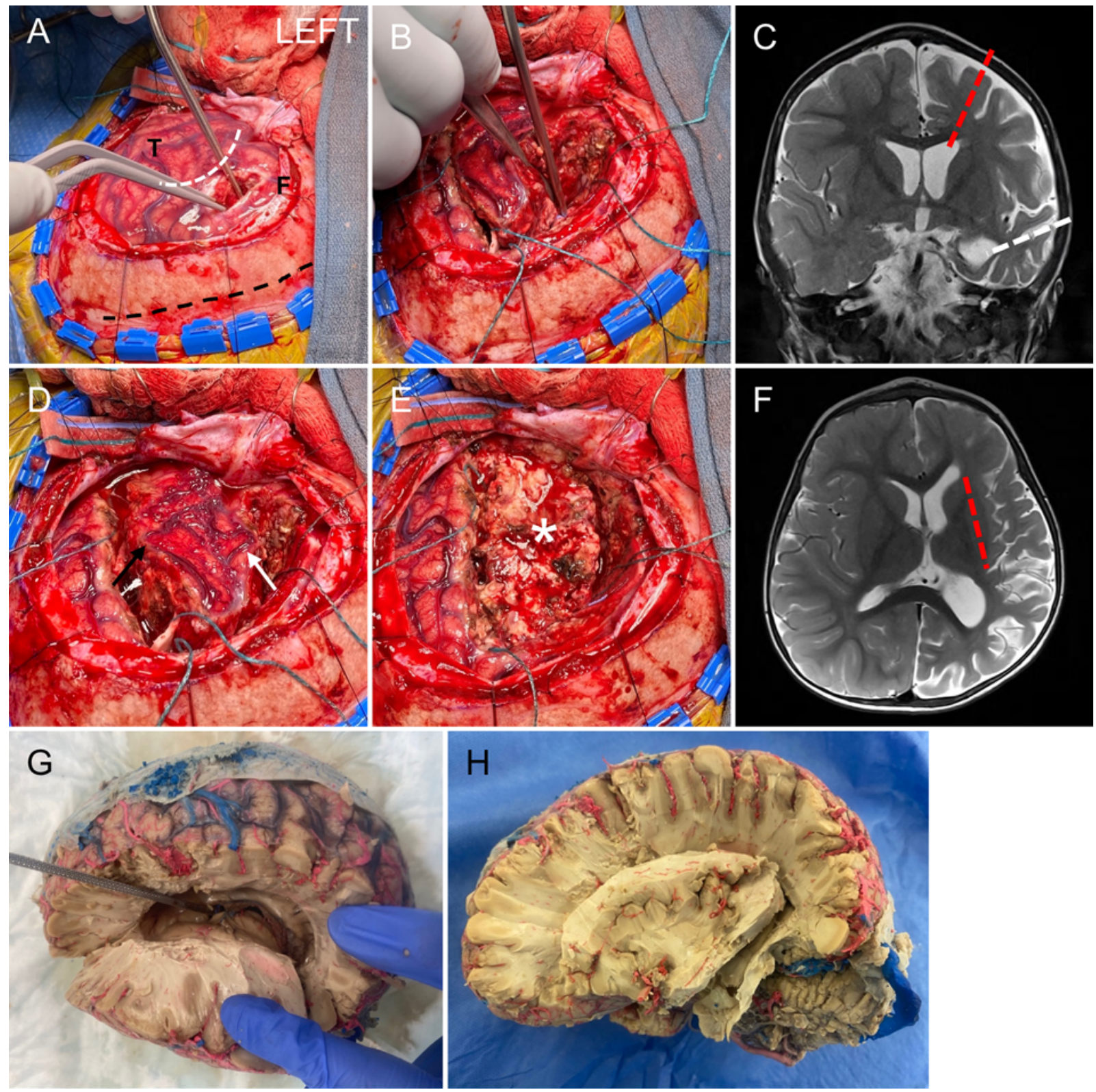

FIG. 3. Lateral disconnection. A: To disconnect the fibers forming the internal capsule and corona radiata, a cortisectomy is performed in the middle frontal gyrus into the frontal lateral horn of the lateral ventricle. The locations of the frontal lobe $(F)$, temporal lobe (T), sylvian fissure (white dotted line), and midline (black dotted line) are shown. B: The cortisectomy is extended posteriorly to the supramarginal gyrus following the course of the lateral ventricle into the atrium and turns inferiorly toward the temporal horn C: Coronal MR image showing the locations of the frontal (red dotted line) and temporal (white dotted line) cortisectomies. D: To facilitate surgical access, the frontal (white arrow) and temporal (black arrow) opercula are resected to expose the insula. E: The insular cortex is resected in a plane, which preserves the basal ganglia structures (asterisk). F: Axial MR image showing the location of the insular resection (red dotted line). G: Cadaveric specimen showing cortisectomy and exposure of the lateral ventricle anteriorly and posteriorly. The dissector is passed through the foramen of Monro. $\mathrm{H}$ : Cadaveric specimen showing removal of the opercula and insula.

tail of the hippocampectomy leads to the atrium and the free tentorial edge. This is connected to the calcar avis and medial wall of the atrium. The white matter dissection is continued superiorly until the falx is reached to complete the parietooccipital disconnection. Anteriorly, the dissection through the rostrum of the corpus callosum is taken medially to the interhemispheric fissure and inferiorly to the anterior skull base. The frontobasal disconnection is completed, and the $A_{1-2}$ junction serves as the posterior limit of the dissection (Fig. 5E-H) to prevent inadvertent damage to the hypothalamus.

\section{Closure}

The surgical cavity is thoroughly irrigated, and an ex- 

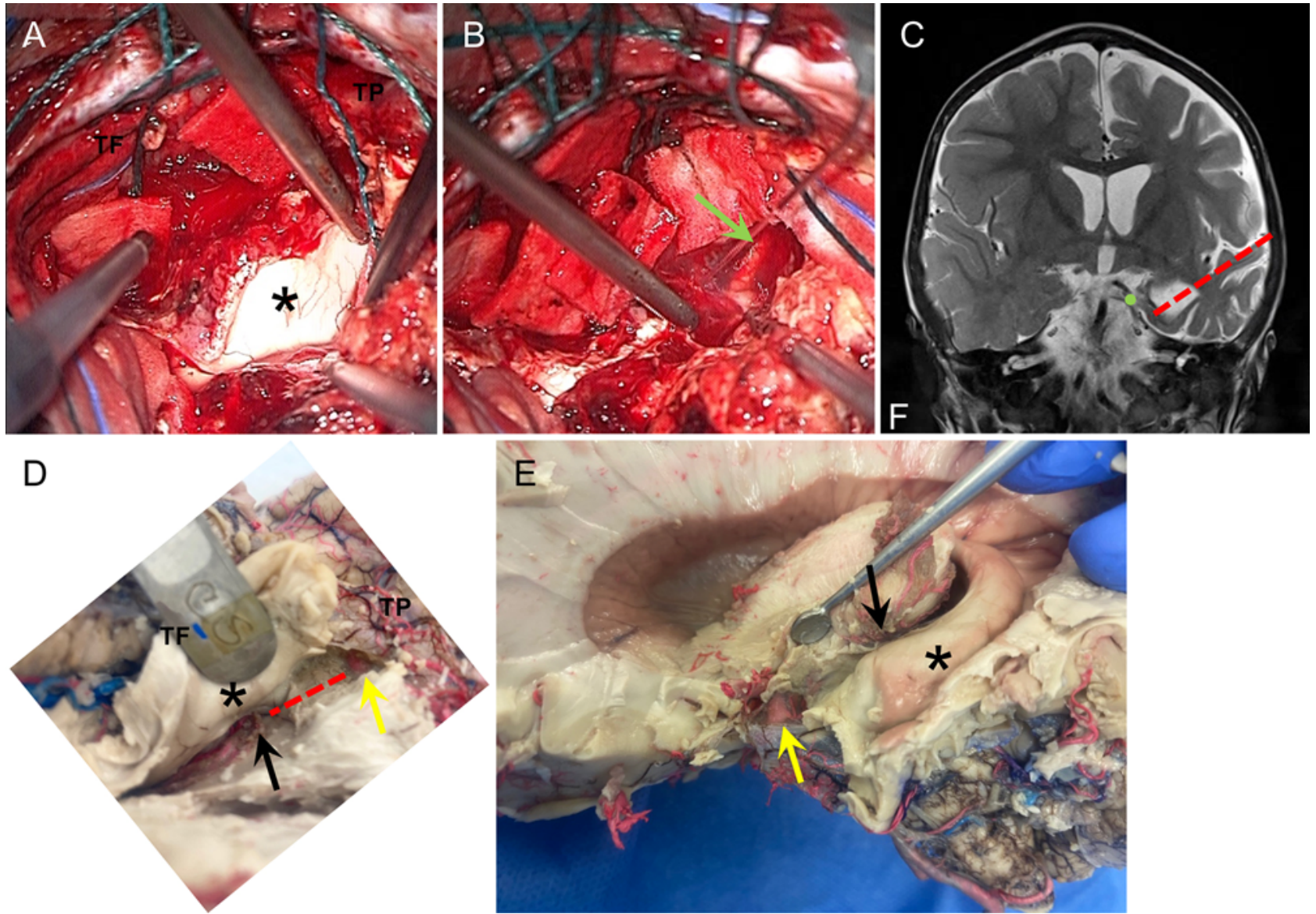

FIG. 4. Mesial temporal resection. A: The temporal horn of the left lateral ventricle is opened to reveal the hippocampus (asterisk) and mesial structures. B: The anterior temporal lobe is resected, paying careful attention to the medial pial plane. When resection is complete, the oculomotor nerve is visualized on the other side of the pia (green arrow). C: Coronal MR image showing the temporal resection (red dotted line) and the relative position of the oculomotor nerve (green dot). D: Cadaveric specimen demonstrating the operative anatomy in panel A. The temporal horn is retracted to show the hippocampus (asterisk). The inferior choroidal point (black arrow) and the MCA bifurcation (limen insulae; yellow arrow) are connected and represent the medial extent of the amygdalectomy (red dotted line). E: The cadaveric specimen is reoriented to show the anatomical position of the hippocampus (asterisk), inferior choroidal point (black arrow), and MCA bifurcation (yellow arrow). TF = temporal floor; TP = temporal pole.

ternal ventricular drain (EVD) is left in situ. The dura is closed primarily and overlaid with a dural substrate. The osteoplastic bone flap is replaced and held in place with resorbable plates and screws (KLS Martin). The skin is closed with absorbable sutures.

\section{Postoperative Care}

The patient is admitted to the intensive care unit after surgery. Postoperative MRI is performed to confirm adequate disconnection (Fig. 6). The EVD is maintained at 5 $\mathrm{cm}$ above the external auditory meatus for 5 days to drain surgical debris in order to prevent hydrocephalus and pseudomeningocele formation. Following discharge, the patient is frequently admitted to inpatient rehabilitation to optimize neurological recovery, with long-term follow-up by the neurodevelopment team. The patient is regularly followed up by the epilepsy team for recurrent seizures and other complications.

\section{Surgical Pearls and Prevention of Complications}

The exact order in which the surgical disconnection is performed is not critical, as long as all parts of the disconnection are adequately carried out. We prefer to resect the insular block early on, as it creates an operative window for the callosotomy and frontobasal and parietooccipital disconnections. There is less tissue to obstruct the operative view and abrogate the use of fixed retractors.

In our practice, the MCA and its branches are taken as necessary with preservation of branches to the frontal and parietal lobes. Some surgeons advocate early ligation of the proximal MCA to reduce operative blood loss, although we have not found a significant difference in operative blood loss with either approach.

Incomplete disconnection leads to persistent or recurrent seizures following hemispherectomy/hemipherotomy. The most frequent culprits are 1) incomplete disconnec- 

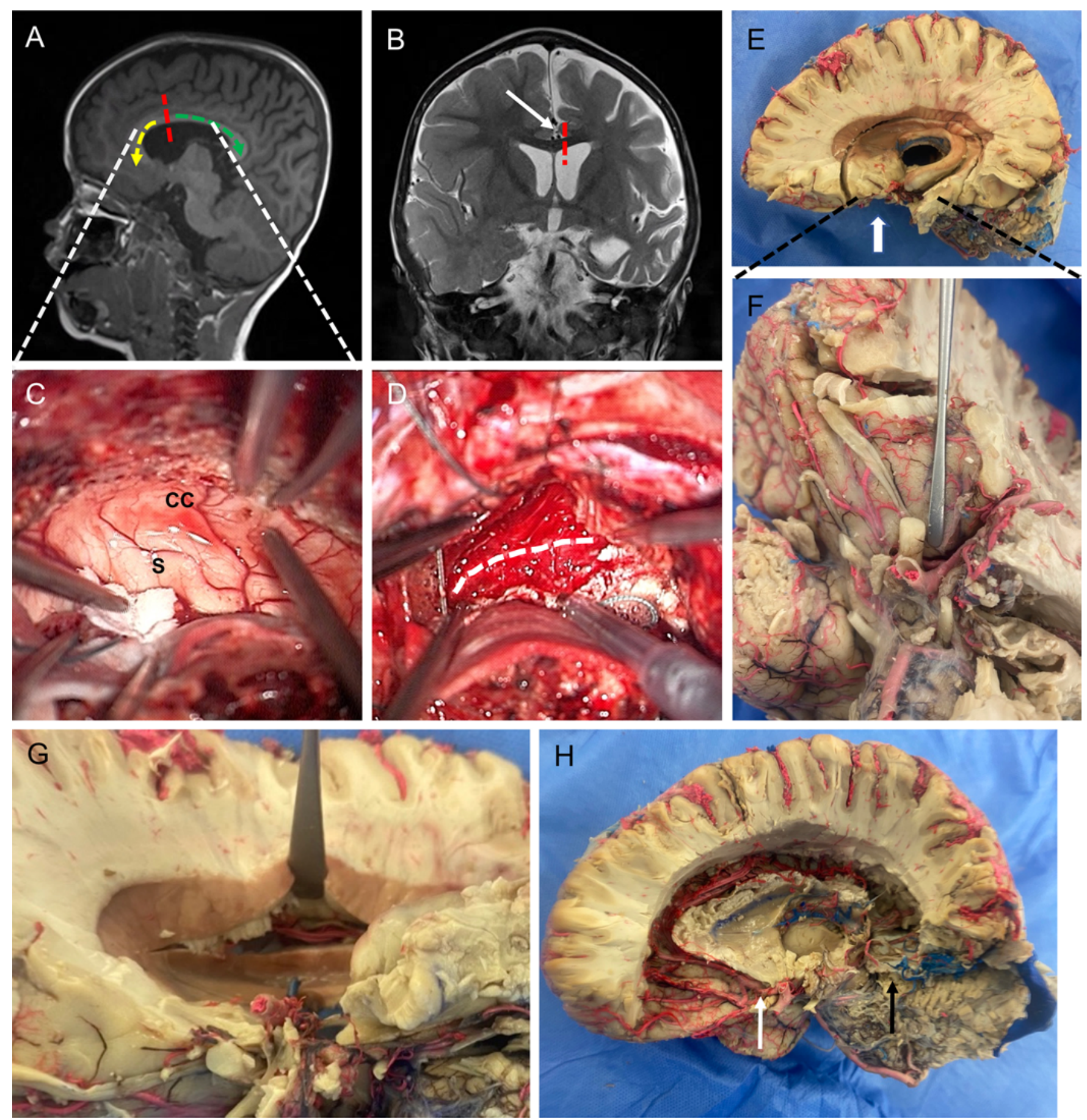

FIG. 5. Corpus callosotomy and completion of disconnections. A and B: Sagittal (A) and coronal (B) MR images showing the corpus callosotomy. The septum pellucidum is identified in the lateral ventricles, and the callosotomy is commenced millimeters lateral to the midline (red dotted lines). Anatomically, pericallosal branches of the ACA and the falx serve as landmarks (white arrow). The callosotomy is taken posteriorly through the body to the splenium until the atrium and the tentorial edge are reached (green dotted arrow). Anteriorly, the dissection follows the ACAs past the genu of the corpus callosum and courses inferiorly and posteriorly through the rostrum (yellow dotted arrow). $\mathbf{C}$ and D: Intraoperative views of the transventricular callostomy. The pericallosal ACA is outlined (white dotted line). CC = corpus callosum; $\mathrm{S}=$ midline septum. $\mathrm{E}$ and $\mathrm{F}$ : Anteriorly, the dissection through the rostrum of the corpus callosum is taken medially to the interhemispheric fissure and inferiorly to the anterior skull base. The frontobasal disconnection is completed, and the $A_{1-2}$ junction serves as the posterior limit of the dissection (tip of the Penfield dissector). The dotted lines and white arrow in panel $\mathrm{E}$ denote the region of interest and direction of view, respectively, in panel F. G: Cadaveric specimen showing the transventricular corpus callostomy and ACA. H: Completion of the frontobasal (white arrow) and the parietooccipital (black arrow) disconnections. The basal ganglia and thalamus have been removed to aid anatomical visualization. 

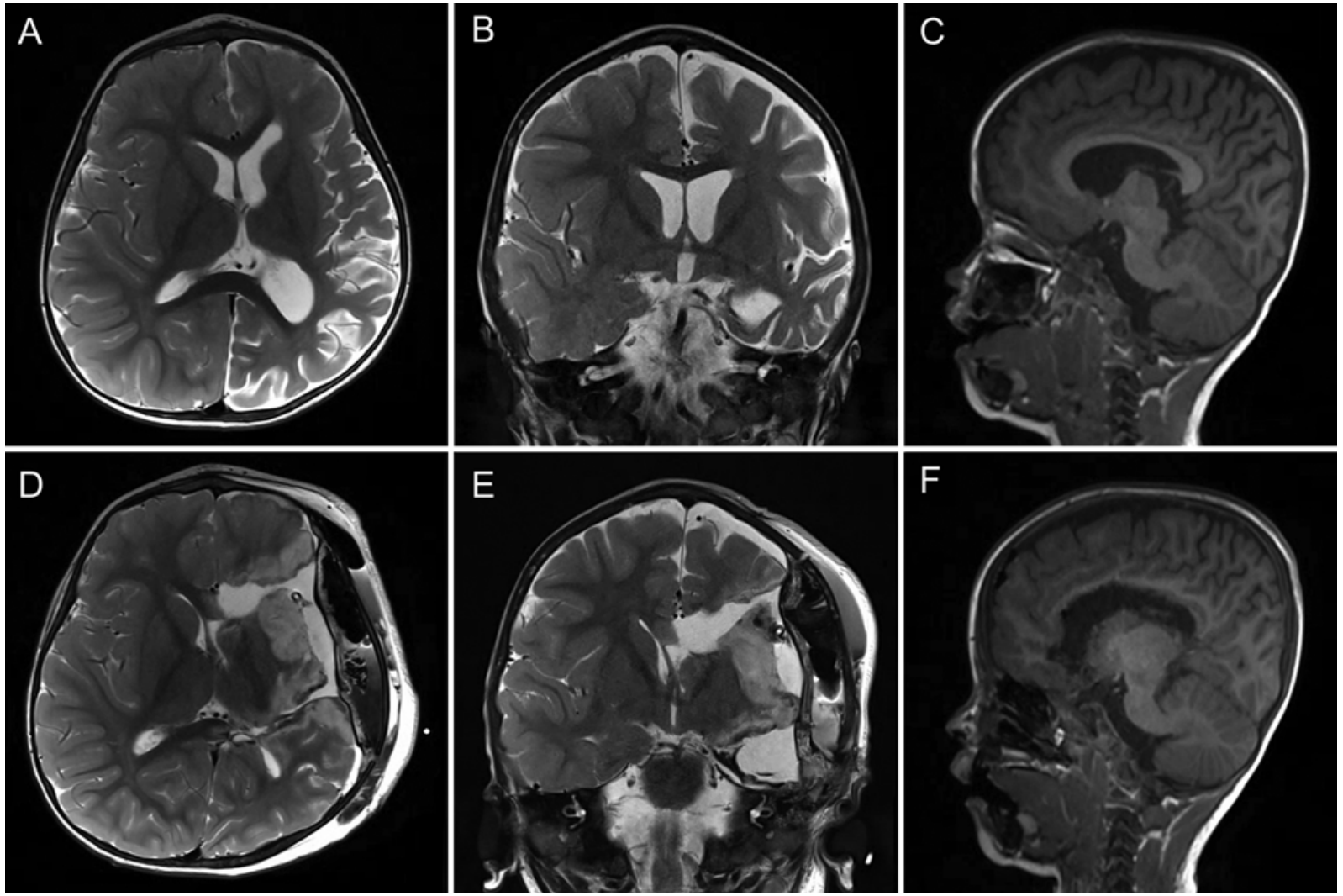

FIG. 6. Anatomical result of functional hemispherectomy/hemispherotomy. Preoperative (A-C) and postoperative (D-F) axial (A and $D$ ), coronal ( $B$ and $E$ ), and sagittal ( $C$ and $F$ ) MR images demonstrating the result of disconnection surgery. Key steps of the disconnection include 1) lateral disconnection of the internal capsule and corona radiata, 2) removal of the opercula and insula, 3) removal of mesial temporal structures, 4) transventricular corpus callostomy, and 5) completion of frontobasal and parietooccipital disconnections.

tion of the corpus callosum, 2) incomplete frontobasal or parietooccipital disconnection, and/or 3) incomplete insular resection. Careful observation of the anatomical landmarks for adequate disconnection and resection is indicated during surgery, with the assistance of neuronavigation. Routine postoperative MRI is performed to confirm adequate disconnection.

Delayed hydrocephalus can manifest in approximately $20 \%$ of patients. Steps taken to reduce the risk of hydrocephalus include placement of a cottonoid at the foramen of Monro after entering the ventricular system to prevent blood products from entering the contralateral ventricles. An EVD is used for several days to promote removal of surgical products and bloody CSF from the ventricular system.

The osteoplastic bone flap preserves the temporalis muscle attachments and blood supply to the bone flap and has been associated with a reduced rate of surgical site infection. ${ }^{2}$ Preservation of the temporalis muscle prevents temporal wasting, and the vascularized bone flap heals and grows along with the pediatric skull to provide a superior cosmetic result.

\section{Surgical and Functional Outcomes}

Functional hemispherectomy/hemispherotomy re- sults in a high rate of seizure control that is comparable to more invasive anatomical hemispherectomy with significantly reduced surgical morbidity. ${ }^{8}$ Limbrick et al. reported a series of 49 patients between the ages of 2 months and 20 years who underwent hemispherotomy by 3 surgeons over 15 years at a single center; 38 patients $(77.6 \%)$ were seizure free at the 2-year follow-up, with significant improvement in seizure control (Engel classes II and III) in the other patients. ${ }^{13}$ In a series of pediatric peri-insular hemispherotomy, Villemure and Daniel reported freedom from disabling seizures (Engel class I) in 34 of 37 patients with adequate follow-up. ${ }^{21}$ In another pediatric series of modified vertical parasagittal hemispherotomy, Delalande et al. reported seizure freedom in $74 \%$ of the 83 children included and significant improvement in all but 2 patients (Engel classes II and III). ${ }^{6}$ Seizure control in vascular infantile hemiplegia and Rasmussen's encephalitis was superior to migrational disorders such as hemimegalencephaly and hemispheric cortical dysplasia. ${ }^{3,6,14,21}$ Over time, however, there was significant decline in seizure control, and Jonas et al. reported a decrease in the seizure-free rate from $78.6 \%$ at 6 months to $58.0 \%$ at 5 years of follow-up. ${ }^{9}$ A review of 186 pediatric cases by Moosa et al. reported a 56\% seizure-free rate at the 6-year follow-up. ${ }^{16}$

After hemispherectomy/hemispherotomy, most patients 
showed improved developmental achievement, with 57\% of patients showing improvement of 5 points or better on the Vineland Adaptive Behavior Scale developmental quotient. ${ }^{9}$ Notably, the degree of improvement varied by seizure substrate, with hemicortical dysplasia showing the most improvement and infarct/ischemia the least improvement, although age difference between the 2 substrate groups was not taken into account. Other factors associated with improved developmental outcome after disconnection surgery include seizure freedom postsurgery and a shorter duration of seizures ( $<3$ years), regardless of seizure substrate. Unsurprisingly, over time, patients who underwent left-sided disconnection had worse functional scores than those who underwent right-sided disconnection. This is likely attributable to the difference between chronological and mental age and the inability of the right hemisphere to develop dominant-hemisphere functions measured by functional scales. Nevertheless, the majority of parents reported improvement in behavior (91\%) and language $(82 \%)$ in children who underwent disconnection. Seventy percent of patients developed satisfactory spoken language skills, with a statistically nonsignificant trend for worse outcome in patients with a younger age at seizure onset and left-sided disconnection. , $^{5,16}$

Motor function usually deteriorates following surgery and improves over months. In a pediatric series, Delalande et al. reported that $84 \%$ of patients were able to eventually walk independently or with assistance, including all children who walked preoperatively. In the paretic upper extremity, $61 \%$ recovered gross motor movements, although none regained fine motor movements. ${ }^{6}$

\section{Conclusions}

Since its inception, hemispheric disconnection has undergone significant modification and improvement from the original anatomical hemispherectomy to the functional hemispherectomy/hemispherotomy performed today. In the pediatric population, in appropriately selected patients, surgical morbidity is low, and the seizure control rate is $>80 \%$ with an excellent scope for functional recovery. A multidisciplinary approach is important, and surgery should be performed at high-volume epilepsy surgery centers for optimal outcome.

\section{Acknowledgments}

We thank Sharon Durfy, PhD, for her assistance with manuscript preparation, and Britta Dedekind, $\mathrm{MBChB}, \mathrm{MMed}$, for her logistical support.

\section{References}

1. Bahuleyan B, Robinson S, Nair AR, Sivanandapanicker JL, Cohen AR: Anatomic hemispherectomy: historical perspective. World Neurosurg 80:396-398, 2013

2. Bass DI, Buckley R, Meyer RM, Lawrence B, Paschall C, Ojemann J, et al: Standard free versus osteoplastic craniotomy: assessment of complication rates during intracranial electroencephalogram electrode placement for seizure localization. World Neurosurg 132:e599-e603, 2019

3. Bulteau C, Otsuki T, Delalande O: Epilepsy surgery for hemispheric syndromes in infants: hemimegalencepahly and hemispheric cortical dysplasia. Brain Dev 35:742-747, 2013

4. Cross JH, Jayakar P, Nordli D, Delalande O, Duchowny M,
Wieser HG, et al: Proposed criteria for referral and evaluation of children for epilepsy surgery: recommendations of the Subcommission for Pediatric Epilepsy Surgery. Epilepsia 47:952-959, 2006

5. Curtiss S, de Bode S, Mathern GW: Spoken language outcomes after hemispherectomy: factoring in etiology. Brain Lang 79:379-396, 2001

6. Delalande O, Bulteau C, Dellatolas G, Fohlen M, Jalin C, Buret V, et al: Vertical parasagittal hemispherotomy: surgical procedures and clinical long-term outcomes in a population of 83 children. Neurosurgery 60 (2 Suppl 1):ONS19ONS32, 2007

7. Engel J Jr: Surgery for seizures. N Engl J Med 334:647-652, 1996

8. Hu WH, Zhang C, Zhang K, Shao XQ, Zhang JG: Hemispheric surgery for refractory epilepsy: a systematic review and meta-analysis with emphasis on seizure predictors and outcomes. J Neurosurg 124:952-961, 2016

9. Jonas R, Nguyen S, Hu B, Asarnow RF, LoPresti C, Curtiss $\mathrm{S}$, et al: Cerebral hemispherectomy: hospital course, seizure, developmental, language, and motor outcomes. Neurology 62:1712-1721, 2004

10. Kim JS, Park EK, Shim KW, Kim DS: Hemispherotomy and functional hemispherectomy: indications and outcomes. J Epilepsy Res 8:1-5, 2018

11. Kovanda TJ, Rey-Dios R, Travnicek J, Cohen-Gadol AA: Modified periinsular hemispherotomy: operative anatomy and technical nuances. J Neurosurg Pediatr 13:332-338, 2014

12. Krynauw RA: Infantile hemiplegia treated by removing one cerebral hemisphere. J Neurol Neurosurg Psychiatry 13:243-267, 1950

13. Limbrick DD, Narayan P, Powers AK, Ojemann JG, Park TS, Bertrand M, et al: Hemispherotomy: efficacy and analysis of seizure recurrence. J Neurosurg Pediatr 4:323-332, 2009

14. Marras CE, Granata T, Franzini A, Freri E, Villani F, Casazza M, et al: Hemispherotomy and functional hemispherectomy: indications and outcome. Epilepsy Res 89:104-112, 2010

15. McKenzie KG: The present status of a patient who had the right cerebral hemisphere removed. JAMA 111:168-183, 1938

16. Moosa ANV, Jehi L, Marashly A, Cosmo G, Lachhwani D, Wyllie E, et al: Long-term functional outcomes and their predictors after hemispherectomy in 115 children. Epilepsia 54:1771-1779, 2013

17. Oppenheimer DR, Griffith HB: Persistent intracranial bleeding as a complication of hemispherectomy. J Neurol Neurosurg Psychiatry 29:229-240, 1966

18. Rasmussen T: Hemispherectomy for seizures revisited. Can J Neurol Sci 10:71-78, 1983

19. Schramm J: Hemispherectomy techniques. Neurosurg Clin N Am 13:113-134, ix, 2002

20. Villemure JG, Mascott CR: Peri-insular hemispherotomy: surgical principles and anatomy. Neurosurgery 37:975-981, 1995

21. Villemure JG, Daniel RT: Peri-insular hemispherotomy in paediatric epilepsy. Childs Nerv Syst 22:967-981, 2006

22. Wada J, Rasmussen T: Intracarotid injection of sodium amytal for the lateralization of cerebral speech dominance: experimental and clinical observations. J Neurosurg 17:266-282, 1960

23. Wen HT, Rhoton AL Jr, Marino R Jr: Anatomical landmarks for hemispherotomy and their clinical application. J Neurosurg 101:747-755, 2004

24. White HH: Cerebral hemispherectomy in the treatment of infantile hemiplegia; review of the literature and report of two cases. Confin Neurol 21:1-50, 1961

25. Wilson PJ: Cerebral hemispherectomy for infantile hemiplegia. A report of 50 cases. Brain 93:147-180, 1970 


\section{Disclosures}

Dr. Hauptman: consultant for Medtronic.

\section{Author Contributions}

Conception and design: Young, Ojemann, Hauptman. Analysis and interpretation of data: Young, Williams, Feroze, McGrath. Drafting the article: Young, Williams, Feroze, McGrath. Critically revising the article: Young, Hauptman. Reviewed submitted ver- sion of manuscript: all authors. Approved the final version of the manuscript on behalf of all authors: Young. Administrative/technical/material support: Ravanpay, Ellenbogen, Hauptman. Study supervision: Ravanpay, Ellenbogen, Ojemann, Hauptman.

\section{Correspondence}

Christopher C. Young: c/o Neurosurgery Publications,

Harborview Medical Center, University of Washington, Seattle, WA.respub@uw.edu. 